\title{
Aneurysm of sinus of Valsalva
}

INSERM

\section{Source}

INSERM. (1999). Orphanet: an online rare disease and orphan drug data base. Aneurysm of sinus of Valsalva. ORPHA:1054

Sinus of Valsalva aneurysm (SVA) is a rare congenital heart malformation of one or more of the aortic sinuses, consisting of a dilation that when unruptured is usually asymptomatic but when ruptured presents with progressive exertional dyspnea, fatigue, chest pain and that can lead to congestive heart failure if left untreated. 